\title{
Improved Spatially Adaptive Denoising Algorithm to Suppress Gaussian Noise in an Image
}

\author{
Sarmila Padhy \\ Department of Computer Science \\ NIT, Rourkela, Odisha, India
}

\author{
Ratnakar Dash \\ Department of Computer Science \\ NIT, Rourkela, Odisha, India
}

\begin{abstract}
In this paper, an improved Spatially Adaptive Denoising Algorithm (SADA) is proposed which leads to satisfactory results in terms of objective and subjective when the image is corrupted with the SNR level less than $10 \mathrm{~dB}$ of additive white Gaussian noise. In general, suppression of Gaussian noise poses a trade-off problem between denoising and preserving the detailed information of the image. So in this proposed method, the parameters of local statistics are used for effective noise suppression with preserving detailed information as compared to PWMAD, SAWM and SADA methods. All pixels including the diagonal elements of the local window with uniform weighting coefficients are taken in our proposed method for the noise detection and removal. Local statistics, computational cost, over-smoothness, error detection and smoothing degree of reconstructed image are the parameters taken into account to effectively remove the noise components in the proposed method.
\end{abstract}

\section{General Terms}

Denoising in Image Processing.

\section{Keywords}

Error detection, denoising, Gaussian noise, local statistics, over-smoothness.

\section{INTRODUCTION}

Digital image processing means the processing of a picture by a digital computer. Lighting and camera properties are the factors which affect an image. Noise is the main factor which degrades the quality of the image. The important features of the image are lost by noise. Noise is introduced into images at the time of transferring and acquiring images [1]. Two noise models can effectively characterize most noise added to images: additive Gaussian noise and Impulsive noise. Gaussian noise is added to the images at the time of image acquisition, whereas impulsive noise is added at the time of transmitting image data over an unsecure communication channel, while it can also be added by acquiring. Gaussian noise is defined as a set of values taken from a zero mean Gaussian distribution which are added to each pixel value, whereas impulsive noise is defined as changing a part of the pixel value with random ones [2]. Enhancing the image quality without loss of features of the image is the main task of denoising. Denoising is the one of the preprocessing stage of the image processing. The number of noise suppression algorithms have been developed. Due to the low computational cost benefits mean filter, median filter and their modified approaches have been usually used. Impulsive noise removal consists of detecting the noisy pixel taking into account the edges and substituting the noisy pixel with the best approximation of the correct pixel value based on the neighborhood, whereas Gaussian noise removal consists of detecting the edges, preserve them for blurring and smoothing the locally smooth and distinct areas. An image represents a degraded version of an original image due to the additive noise which is caused by a noise sensor, recording process, communication channels and any combination of them [3]. In the spatial domain noise estimation algorithms are categorized using two approaches: Block-based approach and Filteringbased approach.

In block-based approach, images are tessellated into a number of blocks. For all the blocks, standard deviations of intensity are computed and then sorted. The block having the smallest standard deviation has the least change of intensity. Although block-based approach is simple, but for small noise it tends to overestimate and for large noise it tends to underestimate. Their estimates may vary significantly depending on the input image and noise levels which is the main difficult task of this approach. Literatures which have followed the block-based approach are described below.

Shin has proposed a block-based noise estimation method, in which an image is filtered by an adaptive Gaussian filter which is corrupted by the additive white Gaussian noise. In this literature, Gaussian filter coefficients are selected as functions of the standard deviation of the Gaussian noise which is estimated from the difference of the selected block images between the noisy input image and its filtered image [4]. Another method for noise level estimation and denoising is proposed by Bosco in which the standard deviation of additive white Gaussian noise in digital image is computed for the selected flat areas which is used to remove Gaussian noise. The problem of estimation of noise level is also addressed [5]. Vijayakumar has proposed a fast and efficient algorithm to remove Gaussian noise in digital images in which the amount of noise level is estimated in the first stage from the degraded image which is corrupted by additive white Gaussian noise. In the next stage, based on a threshold value the central pixel is substituted by the mean value of the surrounding pixels [6].

In filtering-based approach, a low-pass filter is used to filter a noisy input image. The standard deviation between the noisy input image and its filtered image is estimated for images. For large noise cases this approach especially results better estimation of noise. Literatures which have followed the Filtering-based approach are described below.

Crnojevic represents an impulse noise removal based on the pixel-wise median absolute difference (PWMAD) in which a robust estimator of the variance, MAD (median of the absolute deviations from the median), is modified and used to efficiently separate noisy pixels from the image details. This method is free of varying parameters, requires no previous 
training or optimization and successfully removes all types of impulse noise with arbitrary probabilities [7]. In addition, Ibrahim has proposed a switching based adaptive weighted mean filter method, in which the pixels are roughly divided into two classes based on only the intensity values which are noise-free pixel and noisy-pixel. Adaptively changing the size of the median filter can be done based on the number of noisefree pixels in the neighborhood [8]. Nguyen has proposed one technique in which the parameters of local statistics are used for a single image corrupted by Gaussian noise. This method consists of two stages: Noise Detection and Noise Removal Filtering. In noise detection stage, local statistics parameters are used to define the noise detection constraints. In noise removal filtering stage, a modified Gaussian noise removal filter based on the local statistics is defined for controlling the degree of noise suppression because this filter is an adequate way to handle the degree of local smoothness [9].

\section{PROPOSED METHOD}

Generally, when an original image is degraded by additive noise which is signal independent, then the usual degradation model at a point $(i, j)$ can be represented as

$$
O(i, j)=I(i, j)+n(i, j)
$$

where $I$ signifies the original image, $O$ signifies the observed noisy image and $n$ signifies the additive noise which is signal independent respectively.

SADA leads to satisfactory results when the image is corrupted with the SNR level $\geq 10 \mathrm{~dB}$ of additive white Gaussian noise. But when the input image is corrupted seriously with SNR level less than $10 \mathrm{~dB}$, then the SADA does not lead to satisfactory results. So to improve the SADA, a new method is proposed which is described below.

In the proposed method the usual degradation model of the image is considered which is represented in Equation (1). For suppressing the noise from the noisy image, window based concept is used in which the local window of size $(2 U+1) \times$ $(2 \mathrm{~V}+1)$ as shown in Figure (1) is processed for the entire noisy image.

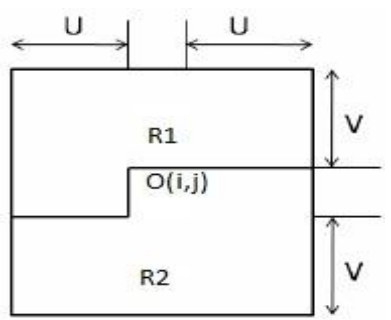

Figure 1: Local Window

Here $\mathrm{U}=1$ and $\mathrm{V}=1$ is considered. The local window is divided into two regions R1 and R2, where R1 represents the dark region and $\mathrm{R} 2$ represents the white region and the intersection between R1 and R2 is null. The filtered pixels belong to the dark region and the observed pixels belong to the white region. As the local statistics parameters are effectively used to control the degree of noise suppression in an image, so the noise detection constraints are defined with the help of the local statistics parameters such as the local weighted mean $\mu_{i, j}$, the local weighted variance $\sigma_{i, j}$ and the local maxima $O_{\max }(i, j)$. For a pixel $O(i, j)$ of the observed portion of the local window, these constraints are computed with the help of the following Equations (2), (3) and (4).

In Equation (2), $w(k, l)$ is the weighting coefficient at the point $(k, l)$, within the window. Now the problem is to filter or reconstruct pixels for the noisy pixels, by taking the pixels of $\mathrm{R} 1$ region and the $\mathrm{R} 2$ region of the local window. By using the local statistics parameters, the pixel $O(i, j)$ of the observed portion of the local window can be detected as noisy or not.

$$
\begin{aligned}
\operatorname{flag}(i, j)=1, & \text { if } O(i, j)>\left(\mu_{i, j}+F_{i, j}\right) \\
& \text { or } O(i, j)<\left(\mu_{i, j}-F_{i, j}\right)
\end{aligned}
$$

0 , otherwise

$$
F_{i, j}=m \times \frac{\sigma_{i, j}}{O_{\max }(i, j)}
$$

where $m$ is a constant value. If the $f l a g(i, j)$ value is equal to 1 , then the pixel is detected as a noisy one. This shows how the local statistics affects the noise detection. A smaller $F_{i, j}$ shows tighter bounds, so the additive noise can be easily detected in the flat region. Here it can be said a higher activity region tends to the looser bounds. This is the agreement of the masking property which is represented in [10]. Then with the help of the Gaussian filter, the noisy pixel is reconstructed. Here, the Gaussian filter is the adequate way for handling the degree of local smoothness since it is defined as a function of local statistics which is very useful to control the degree of the smoothness of the reconstructed image using local activity. The Gaussian filter is:

$h_{i, j}=\frac{1}{S} \exp \left\{-P \frac{\sigma_{i, j}^{2}\left(\sigma^{2}+j^{2}\right)}{\sqrt{\mu_{i, j}+1}}\right\}$

where $S$ denotes the normalizing constant and $P$ denotes a tuning parameter. The parameter $P$ determines the smoothing degree of the reconstructed image. Smaller $P$ leads to stronger low-pass filtering which results over-smoothness around edge information whereas larger $P$ leads to weaker low-pass filtering but suppression of noise is not satisfactory. Now the filtered pixel for the noisy pixel is defined in Equation (8).

In the proposed method, it is considered that for relatively high level of noise means when the input image is corrupted seriously with SNR level less than $10 \mathrm{~dB}$, then by using the Equation (5), the pixel is detected as noisy. Then that noisy pixel is filtered by using the Equations (7) and (8). Here the noise detection and removal methods are implemented using all pixels including the diagonal elements of the local window with uniform weighting coefficients. Otherwise for any level of SNRs, the noise detection and removal methods are 
$\mu_{i, j}=\frac{\sum_{k} \sum_{l,(k, l) \varepsilon R 1} w(k, l) x^{\prime}(i+k, j+l)+\sum_{k} \sum_{l,(k, l) \varepsilon R 2} w(k, l) O(i+k, j+l)}{\sum_{k} \sum_{l,(k, l) \varepsilon R 1} w(k, l)+\sum_{k} \sum_{l,(k, l) \varepsilon R 2} w(k, l)}$

$\sigma_{i, j}=\frac{\sum_{k} \sum_{l,(k, l) \varepsilon R 1} w(k, l)\left|x^{\prime}(i+k, j+l)-\mu_{i, j}\right|+\sum_{k} \sum_{l,(k, l) \varepsilon R 2} w(k, l)\left|O(i+k, j+l)-\mu_{i, j}\right|}{\sum_{k} \sum_{l,(k, l) \varepsilon R 1} w(k, l)+\sum_{k} \sum_{l,(k, l) \varepsilon R 2} w(k, l)}$

$O_{\max }(i, j)=\max \left(\max _{(k, l) \varepsilon R 1} x^{\prime}(k, l), \max _{(k, l) \varepsilon R 2} O(k, l)\right)$

$x_{i, j}^{\prime}=\frac{\sum_{k} \sum_{l,(k, l) \varepsilon R 1} h(k, l) x^{\prime}(i+k, j+l)+\sum_{k} \sum_{l,(k, l) \varepsilon R 2} h(k, l) O(i+k, j+l)}{\sum_{k} \sum_{l,(k, l) \varepsilon R 1} h(k, l)+\sum_{k} \sum_{l,(k, l) \varepsilon R 2} h(k, l)}$

implemented as per SADA without using the diagonal pixels along with nonuniform weighting coefficients. This process is repeated until the local window is processed for the entire input image.

\section{RESULTS AND DISCUSSIONS}

The proposed denoising algorithm is tested with various standard gray-level images like Cameraman, Lena, Goldhill, Monarch and bird of size $256 \times 256,8$-bits/pixel corrupted by Gaussian noise of various levels of SNRs and compared with some standard methods like PWMAD, SAWM and SADA. The performance of the proposed method is measured by the parameter peak signal-to-noise ratio (PSNR) which is defined as:

$$
P S N R=10 \log 10 \frac{255^{2}}{\frac{1}{M \times N} \sum_{i}^{M} \sum_{j}^{N}\left(R_{i, j}-I_{i, j}\right)^{2}}
$$

Where, $R_{i, j}$ and $I_{i, j}$ represents the pixel values of the restored image and the original image respectively and $M \times N$ is the size of the image. Figures (2) and (3) show the reconstructed images for the Cameraman image and the Lena image degraded with $5 \mathrm{~dB}$ of Gaussian noise respectively. The PSNR comparisons of the same images for various SNR levels are shown in Table 1 and Table 2. It is observed that in this experiment, when the noise level is relatively high, i.e. less than $10 \mathrm{~dB}$, then PWMAD, SAWM and SADA leads to overly blurred results whereas the proposed method leads to relatively satisfactory results in effective noise suppression with preserving detailed informations. But when the noise level is low i.e. $\geq 10 \mathrm{~dB}$, then the proposed method leads to similar results as SADA.

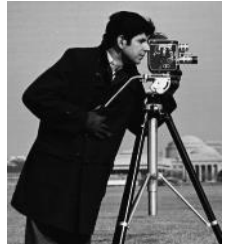

(a)

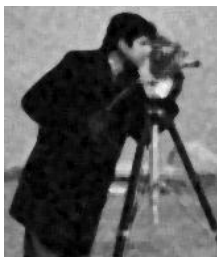

(d)

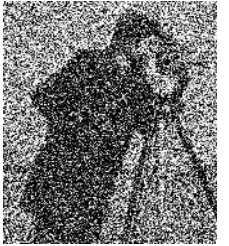

(b)

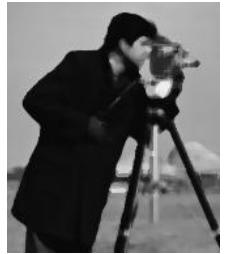

(e)

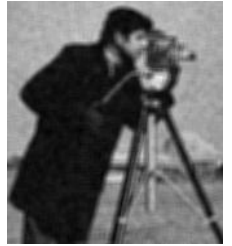

(c)

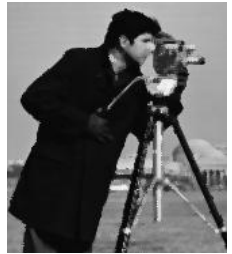

(f)
Figure 2 : Experimental results with Cameraman image: (a) original Cameraman image, (b) noisy image with $5 \mathrm{~dB}$ of Gaussian noise, (c) corresponding reconstructed image with PWMAD, (d) corresponding reconstructed image with SAWM, (e) corresponding reconstructed image with SADA, (f) corresponding reconstructed image with Proposed Method.

Table 1. PSNR Comparisons of Cameraman Image

\begin{tabular}{|c|cccc|}
\hline NOISE & \multicolumn{4}{|c|}{ METHODS } \\
\hline & PWMAD & SAWM & SADA & Proposed \\
\hline $30(\mathrm{~dB})$ & 27.32 & 27.70 & 30.31 & 30.31 \\
\hline $20(\mathrm{~dB})$ & 27.06 & 27.38 & 29.77 & 29.77 \\
\hline $10(\mathrm{~dB})$ & 25.08 & 25.93 & 28.11 & 28.11 \\
\hline $5(\mathrm{~dB})$ & 23.01 & 23.83 & 26.21 & 27.35 \\
\hline
\end{tabular}




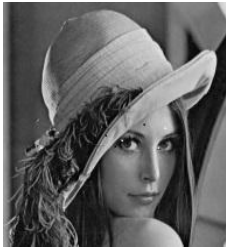

(a)

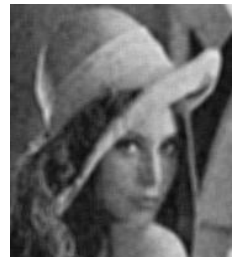

(d)

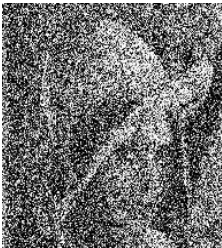

(b)

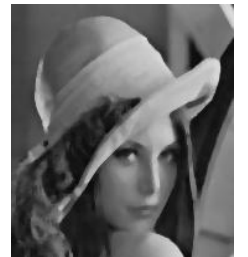

(e)

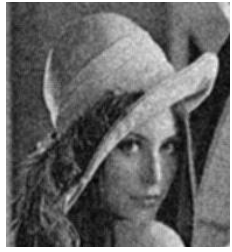

(c)

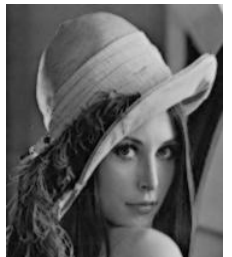

(f)
Figure 3 : Experimental results with Lena image: (a) original Lena image, (b) noisy image with $5 \mathrm{~dB}$ of Gaussian noise, (c) corresponding reconstructed image with PWMAD, (d) corresponding reconstructed image with SAWM, (e) corresponding reconstructed image with SADA, (f) corresponding reconstructed image with Proposed Method.

Table 2. PSNR Comparisons of Lena Image

\begin{tabular}{|c|cccc|}
\hline NOISE & \multicolumn{4}{|c|}{ METHODS } \\
\hline & PWMAD & SAWM & SADA & Proposed \\
\hline $30(\mathrm{~dB})$ & 31.24 & 31.90 & 33.09 & 33.09 \\
\hline $20(\mathrm{~dB})$ & 30.64 & 30.95 & 32.36 & 32.36 \\
\hline $10(\mathrm{~dB})$ & 27.77 & 27.84 & 29.45 & 29.45 \\
\hline $5(\mathrm{~dB})$ & 25.05 & 25.73 & 27.01 & 28.52 \\
\hline
\end{tabular}

\section{CONCLUSIONS}

It is observed that the proposed method provides satisfactory results as compared to PWMAD, SAWM and SADA methods for the highly corrupted image with SNR level of less than $10 \mathrm{~dB}$. This method results in effective noise suppression with preserving detailed information. The reconstructed images with other methods lead to loss of edge information. Also it is observed that the degree of over smoothness is more visible with the other approaches. As the local activity is effectively used to control the degree of noise suppression, so the proposed method leads to relatively satisfactory results. It is verified that due to its simplicity this algorithm requires very low computational cost. In the proposed method, the uniform weighting coefficients and all pixels including the diagonal pixels within the local window are the important parameters used to calculate the local information as well as to obtain a better filtering performance.

\section{REFERENCES}

[1] R. C. Gonzalez and R. E. Woods, Digital Image Processing, Addison Wesley, 1992.

[2] H. C. Andrew and B.R. Hunt, Digital Image Restoration, Prentice Hall, 1977.

[3] T. A. Nodes and N. C. Gallagher, Median filters: some modifications and their properties, in IEEE Trans. Acoustics, Speech and Signal Processing, vol. 30, no. 5, pp. 739--746, 1982.

[4] D. H. Shin, R. H. Park, S. J. Yang, Block based noise estimation using adaptive Gaussian filtering, in IEEE Transaction Consumer Electronics, vol. 51, no. 1, 2005.

[5] A. Bosco, A. Bruna, G. Messina, Fast Method for Noise Level Estimation and Integrated Noise Reduction, in IEEE Transaction On Consumer Electronics, vol. 51, no. 3, pp. 1028--1033, 2005.

[6] V. R. Vijayakumar, P. T. Vanathi, Fast and Efficient Algorithm to Remove Gaussian Noise in Digital Images, in IAENG International Journal of Computer Science, vol. 37 , no. $1,2009$.

[7] V. Crnojevic and V. Senk and Z. Trpovski, Advanced impulse detection based on pixel-wise MAD, in IEEE Signal Processing Letters, vol. 11, no. 7, pp. 589--592, 2004.

[8] H. Ibrahim, N. S. P. Kong, T. F. Ng, Simple adaptive median filter for the removal of impulse noise from highly corrupted images, in IEEE Transaction On Consumer Electronics, vol. 54, no. 4, 2008.

[9] T. Nguyen, W. Song and M. Hong, Spatially adaptive denoising algorithm for a single image corrupted by Gaussian noise, in IEEE Transaction On Consumer Electronics, vol. 56, no. 3, 2010.

[10] G. L. Anderson, A. N. Netravali, image restoration based on a subjective criterion, in IEEE Transactions Systems, Man, and Cybernetics, vol. 6, no. 12, pp. 845-853,1976 